\title{
Micronutrient deficiencies among children and women in Bangladesh: progress and challenges
}

\author{
Faruk Ahmed ${ }^{1} *$, Noreen Prendiville ${ }^{2}$ and Anuradha Narayan ${ }^{3}$ \\ ${ }^{1}$ Public Health, School of Medicine and Menzies Health Institute Queensland, Griffith University, Gold Coast Campus, QLD 4220, Australia \\ ${ }^{2}$ UNICEF, Kampala, Uganda \\ ${ }^{3}$ UNICEF, Dhake, Bangladesh
}

(Received 10 June 2016 - Final revision received 13 August 2016 - Accepted 5 September 2016)

Journal of Nutritional Science (2016), vol. 5, e46, page 1 of 12

doi:10.1017/jns.2016.39

Abstract

This paper provides a comprehensive review of the current situation regarding micronutrient deficiencies among children and women in Bangladesh. This review also discusses the successes and current challenges of existing intervention programmes. Data from nationally representative and selected small surveys since the 1980s that have reported on the status of at least one micronutrient in children and/or women have been examined. National policy documents/reports on existing interventions have been analysed. While the severity of various micronutrient deficiencies has declined since the 1980 s, a significant proportion of preschool-age children remains with deficiencies in vitamin A (20.5 \%), Zn (44.5\%) and vitamin D (39.6\%); about onethird of these children are anaemic, and $10.7 \%$ of the children are Fe deficient. A high proportion of non-pregnant and non-lactating women is deficient in $\mathrm{Zn}(57 \%)$ and I (42\%), while one-quarter of women live with anaemia and vitamin $\mathrm{B}_{12}$ and vitamin $\mathrm{D}$ (21 \%) deficiencies. Nearly one-half of the pregnant and lactating women are anaemic. Suboptimal diets, poor hygiene, infection and infestation are identified as some of the key factors associated with high levels of deficiencies. Multiple approaches and interventions are being supported, and while some notable progress has been achieved, significant challenges continue, including those related to coverage, quality and compliance. It is concluded that although current intervention programmes have made some progress in controlling the severe deficiencies, micronutrient deficiencies in Bangladesh remain a considerable problem. More well-integrated approaches for strengthening the existing intervention programmes are needed. In addition, new intervention strategies for alleviating and preventing specific micronutrient deficiencies are recommended.

Key words: Micronutrients: Anaemia: Intervention programmes: Policy: Bangladesh

Micronutrient deficiency is widely prevalent throughout the world, affecting more than two billion people ${ }^{(1)}$. However, the scale of the problem is much greater in low- and middle-income countries where multiple micronutrient deficiencies often present concomitantly as a result of diets with limited diversity, poor bioavailability and limited micronutrient content, in addition to poor hygiene and infections ${ }^{(2)}$. Micronutrient deficiencies (i.e. vitamin $\mathrm{A}$ and $\mathrm{Zn}$ deficiencies, in particular) have been estimated to account for one million child deaths per year, and Fe deficiency alone is responsible for 115000 maternal deaths per year ${ }^{(3)}$. In addition to an increased risk of mortality, micronutrient deficiencies are associated with impaired resistance to infections, decreased nutrient uptake and delayed or impaired physical, mental and psychomotor development ${ }^{(4,5)}$. Therefore, these deficiencies affect people's quality of life, which can have an impact on productivity $^{(1,6)}$. The importance of reducing micronutrient deficiencies in children and women is well recognised.

Abbreviations: BDHS, Bangladesh Demographic and Health Survey; IDA, Fe-deficiency anaemia; IFA, Fe-folic acid; NMS 2011-2012, National Micronutrients Status Survey 2011-2012; MNP, multiple micronutrient powder; NPNL, non-pregnant and non-lactating; VAD, vitamin A deficiency.

*Corresponding author: F. Ahmed, email f.ahmed@griffith.edu.au 
For example, a systematic review of vitamin A supplementation trials in children aged 6-59 months showed a $24 \%$ reduction in all-cause mortality and a $28 \%$ reduction in diarrhoearelated mortality ${ }^{(7)}$. Another review showed that preventive $\mathrm{Zn}$ supplementation in children younger than 5 years reduced diarrhoea by $13 \%$ and pneumonia by $19 \%{ }^{(8)}$. More recently, a systemic review of multiple micronutrient supplementation during pregnancy showed a significant reduction in low birth weight, small-for-gestation and preterm births ${ }^{(9)}$.

In Bangladesh, the prevalence of undernutrition has been decreased significantly and is likely to achieve the nutrition Millennium Development Goal ${ }^{(10)}$. Although several strategies have been implemented over the past decades to address the situation, the prevalence of micronutrient deficiency still remains very high and is considered a significant public health problem ${ }^{(11)}$. The recently concluded National Micronutrients Status Survey 2011-2012 (NMS 2011-2012) $)^{(11)}$, together with the analysis and discussions related to the development of a strategy to address micronutrient deficiencies, has presented an opportunity to examine the current micronutrient status among children and women in the country and review the successes and challenges of existing interventions. This paper also discusses the proposed intervention strategies for the prevention and control of micronutrient deficiencies in Bangladesh.

\section{Methodology}

This review examined all of the available nationally representative surveys since the 1980s that have reported on the prevalence of at least one micronutrient deficiency in children (infants, preschool-age children, school-age children and/or adolescents) and/or women (non-pregnant and non-lactating (NPNL), pregnant and/or lactating women). Data from some small studies were used if national data were not available. Reports on the effectiveness of existing intervention programmes and various national policy documents have been analysed. National surveys and policy documents were searched online using official websites of relevant research institutes/organisations. In addition, the lead research institutes/organisations were consulted to obtain documents that were not available online. The national surveys that did not report on the prevalence of anaemia and/or micronutrient deficiency in children and/or women were excluded. Because of the limited data on $\mathrm{Fe}, \mathrm{Zn}$ and vitamin $\mathrm{B}_{12}$ deficiencies, selected non-representative studies were also included in the analyses.

\section{Current situation of micronutrient deficiency}

This section focuses on the extent and nature of micronutrient deficiencies among children and women in the country and is followed by an analysis of the factors contributing to the deficiency. The literature search identified nine national surveys and four nutrition surveillance reports that met the inclusion criteria. Of the included studies, four reported on the prevalence of vitamin A deficiency (VAD), five on the prevalence of anaemia and three on the prevalence of I deficiency only.
Only one study reported on the prevalence of anaemia and a range of micronutrient deficiencies. The details of the methods and prevalence of various micronutrient deficiencies of the referenced survey reports/articles are summarised in Table 1.

\section{Vitamin A deficiency}

VAD has been identified as a serious public health problem in Bangladesh since the 1960s. Although the severity of the problem has decreased remarkably, the prevalence of VAD (defined by the WHO as a serum retinol concentration $<0.70 \mu \mathrm{mol} / \mathrm{l})$ has remained the same over the past decade, especially among preschool-age (6-59 months) and schoolage children ${ }^{(11)}$. Currently, $20.5 \%$ of the preschool-age children are suffering from VAD, with the highest prevalence among children living in the slums (e.g. $38 \%$ in preschoolage children and $27 \%$ in school-age children) ${ }^{(11)}$. While there are no recent estimates available on the severe forms of VAD, previous surveys indicated that the prevalence of night blindness in preschool-age children has declined remarkably over the past two decades ${ }^{(12,13)}$; it was $3.7 \%$ in 1982 $1983^{(12)}$ and dropped to $0.04 \%$ in $2005^{(13)}$.

The recent NMS 2011-2012 reported only a 5.4\% prevalence of VAD among NPNL women ${ }^{(11)}$, which is similar to the prevalence reported in a 1997-1998 National Vitamin A Survey ${ }^{(14)}$. However, nearly one-third of the NPNL women have a suboptimal vitamin A status (i.e. serum retinol concentration $<1.05 \mu \mathrm{mol} / \mathrm{l})^{(11)}$. No current estimates are available on the prevalence of VAD among pregnant and lactating women.

Low socio-economic status, being a slum dweller, low levels of knowledge of vitamin A-rich food and/or their health benefits, poor intake of animal sources of vitamin A especially by households with severe food insecurity and predominant share of plant-based vitamin A with low bioavailability have been identified as factors associated with VAD among all population groups ${ }^{(11,15)}$.

\section{lodine deficiency}

The current prevalence of I deficiency, based on urinary I concentration $<100 \mu \mathrm{g} / \mathrm{l}$, in school-age children is as high as $40 \%{ }^{(11)}$, reflecting a figure similar to the $42.5 \%$ reported in $1999^{(16)}$ and an increase from the $33.8 \%$ reported in 2004 2005 (Table 1) ${ }^{(17)}$. There are no estimates on the current prevalence of goitres (a severe form of I deficiency) in school-age children. However, previous surveys indicated a substantial reduction in goitre prevalence in school-age children from $50 \%$ in $1993^{(18)}$ to $17 \cdot 2 \%$ in $1999^{(16)}$ to $6.2 \%$ in $2004-2005^{(17)}$.

The NMS 2011-2012 reported a $42 \%$ prevalence of I deficiency, based on urinary I level, in NPNL women ${ }^{(11)}$, which was $45.6 \%$ in $1999^{(16)}$ and $38.6 \%$ in $2004-2005^{(17)}$. The National Iodine Deficiency Disorder Survey in 2004-2005 reported goitre rates of 11.7 and $14.6 \%$ in NPNL women and pregnant women, respectively ${ }^{(17)}$, with the higher risk of 


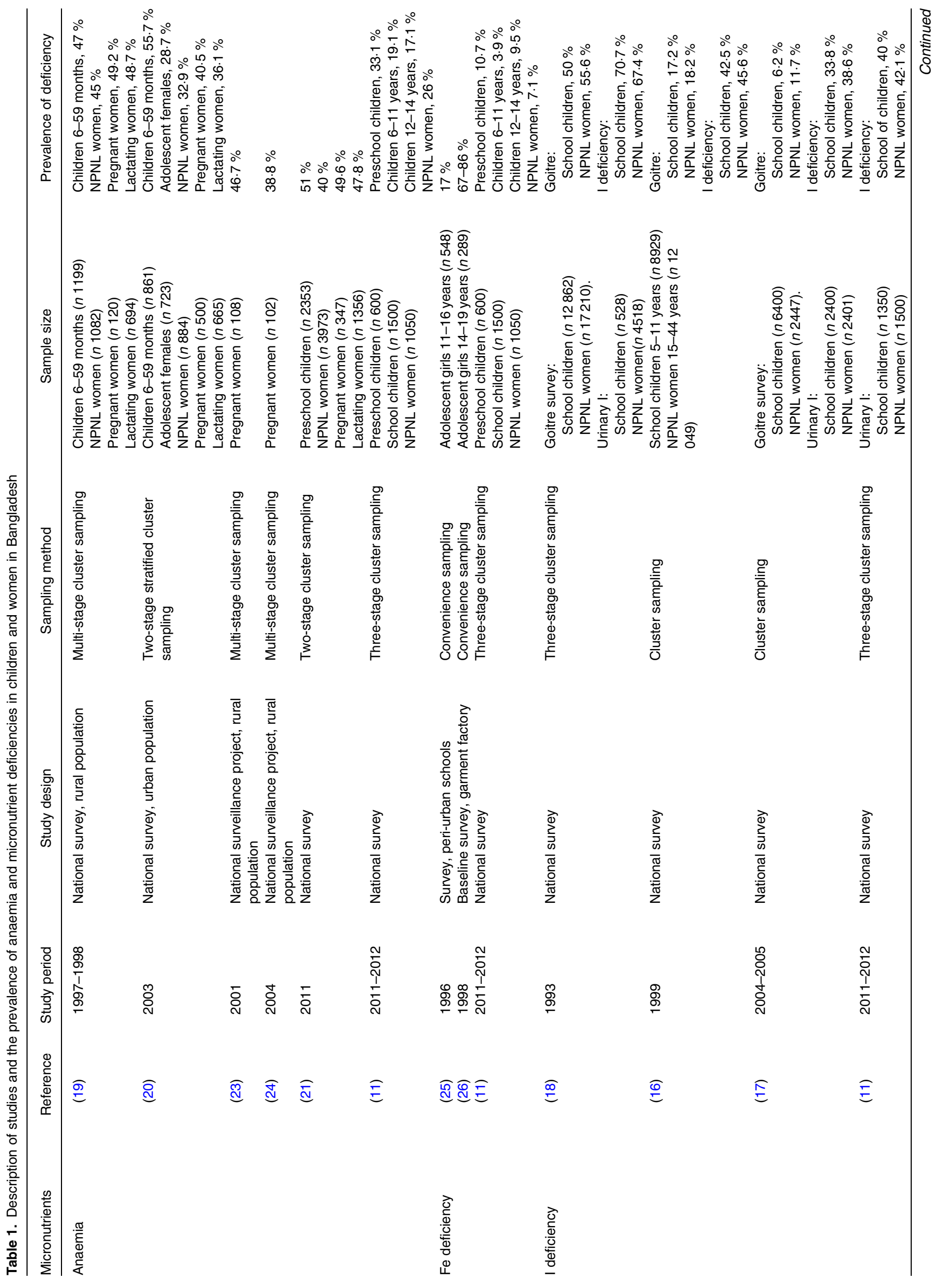




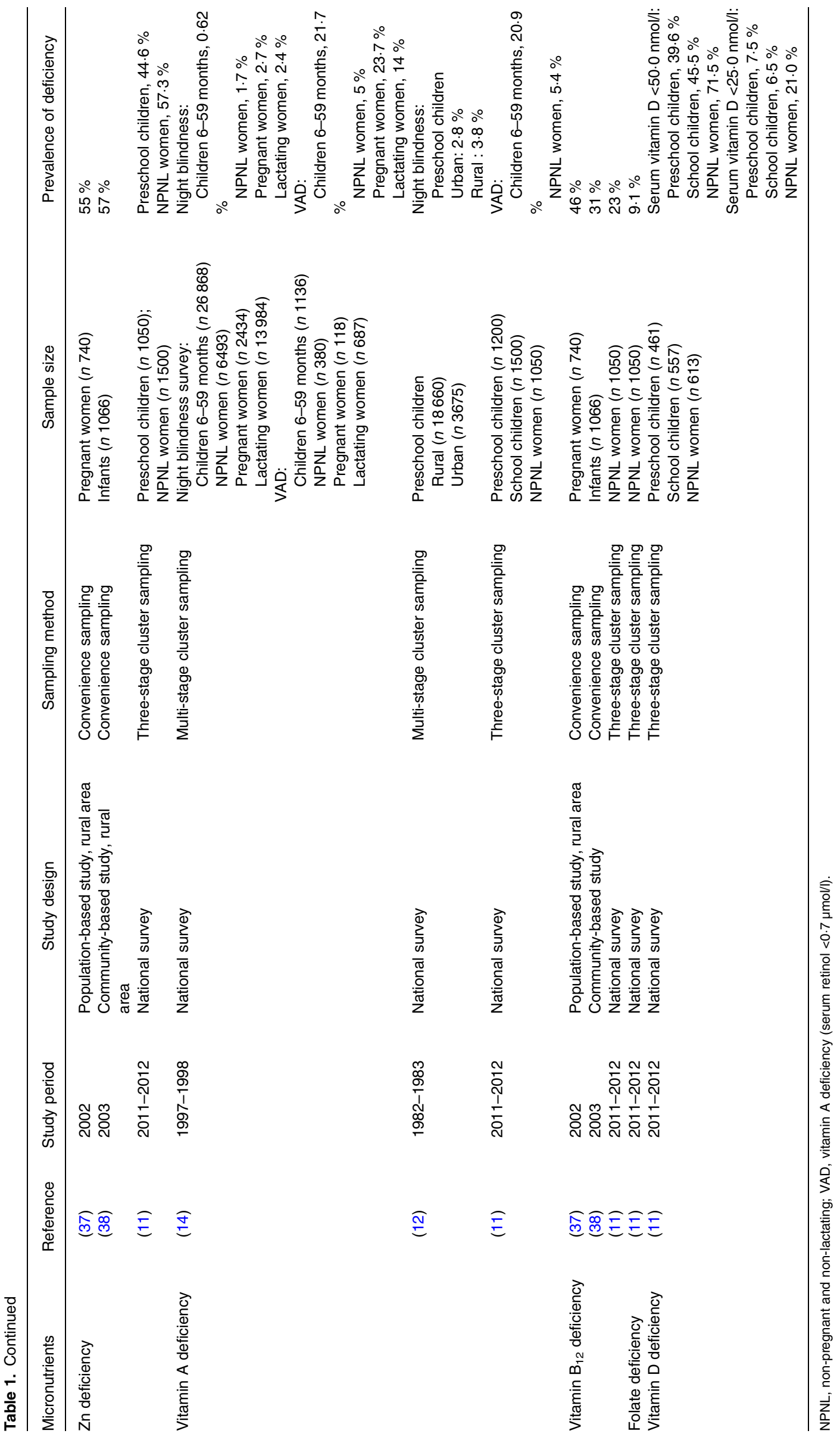


I deficiency among women either living in rural areas and/or belonging to lower socio-economic groups ${ }^{(11)}$.

Household food insecurity, lack of access to iodised packaged salt, rural residency, low levels of awareness about the health benefits of I and iodised salts, consumption of industrial salt (non-iodised) and lack of preservation knowledge about iodised salts have been identified as the major risk factors of I deficiency in the Bangladeshi population ${ }^{(15)}$.

\section{Anaemia and iron deficiency}

According to the NMS 2011-2012, the prevalence of anaemia, defined by an $\mathrm{Hb}$ concentration $<120 \mathrm{~g} / 1$ in NPNL women and $<110 \mathrm{~g} / \mathrm{l}$ in children aged $6-59$ months, affects $33 \%$ of children aged 6-59 months and $26 \%$ of NPNL women ${ }^{(11)}$. These percentages reflect a significant decrease in the prevalence reported in 1997-1998 (47\% in children aged 6-59 months and $45 \%$ in NPNL women) $)^{(19)}$ and in 2003 $(55.7 \%$ in children aged 6-59 months and $32.9 \%$ in NPNL women) ${ }^{(20)}$. However, the Bangladesh Demographic and Health Survey (BDHS) conducted in 2011 found higher levels, with a $51 \%$ prevalence of anaemia in preschool-age children and a $40 \%$ prevalence in NPNL women ${ }^{(21)}$. The NMS 2011-2012 $2^{(11)}$ and BDHS 2011 $1^{(21)}$ were conducted within a 1-year period, but the differences in prevalence are probably attributed to methodological differences. To measure $\mathrm{Hb}$ levels, the BDHS used capillary blood samples by finger prick, whereas the NMS 2011-2012 used venous blood samples. Capillary blood samples often produce inconsistent results (blood drawing requires extreme care and training), whereas venous blood samples produce more reliable results. However, it is clear that a significant proportion of preschool-age children and NPNL women are still anaemic. While there are no recent data, earlier surveys have indicated a $30-40 \%$ prevalence of anaemia among adolescent girls ${ }^{(19-20,22)}$. Further, according to the BDHS in 2011, approximately one-half of the pregnant $(49.6 \%)$ and lactating $(48 \%)$ women are also anaemic ${ }^{(21)}$. In 2001 , the prevalence of anaemia in pregnant women was $47 \%{ }^{(23)}$, but it was $39 \%$ in $2004^{(24)}$, reflecting an increase in the current prevalence among pregnant women.

Earlier small-scale studies indicated that Fe deficiency is a major cause of anaemia ${ }^{(25,26)}$, but the results from the NMS 2011-2012 suggest that the national prevalence of Fe deficiency (serum ferritin concentration $<120 \mu \mathrm{g} / 1$ in preschoolage children and $<150 \mu \mathrm{g} / \mathrm{l}$ in NPNL women) is only $10 \cdot 7$ $\%$ in preschool-age children and $7 \cdot 1 \%$ in NPNL women ${ }^{(11)}$. Further, only $7.2 \%$ of the preschool-age children and $4.8 \%$ of the NPNL women reported suffering from Fe-deficiency anaemia (IDA). IDA was defined by combined cut-offs for an $\mathrm{Hb}$ concentration $<110 \mathrm{~g} / \mathrm{l}$ and serum ferritin $<120 \mu \mathrm{g} / \mathrm{l}$ in preschool-age children and an $\mathrm{Hb}$ concentration $<120 \mathrm{~g} / 1$ and serum ferritin $<150 \mu \mathrm{g} / \mathrm{l}$ in NPNL women ${ }^{(11)}$. Unfortunately, there are no data on Fe deficiency and IDA among pregnant women. The prevalence of IDA is even lower in school-age children ${ }^{(11)}$, indicating that Fe deficiency may not be the major cause of anaemia in the Bangladeshi population. While the NMS 2011-2012 reports that dietary
Fe provides only $41-82 \%$ of the RDA across different population groups ${ }^{(11)}$, Fe content in groundwater appears to be high in some parts of Bangladesh ${ }^{(27)}$, predominantly in the ferrous $\left(\mathrm{Fe}^{2+}\right)$ form, which is more bioavailable than the ferric form ${ }^{(27,28)}$. The low prevalence of Fe deficiency in the NMS 2011-2012 was attributed to the high Fe concentration in drinking water from tube-wells ${ }^{(11)}$. Of note, a small-scale study in northern rural Bangladesh reports a positive association of daily $\mathrm{Fe}$ intake from drinking water with plasma ferritin and total body $\mathrm{Fe}$ in women ${ }^{(29)}$. However, a recent article based on NMS 2011-2012 data reported a differential prevalence of anaemia and Fe deficiency in Bangladeshi NPNL women living in areas of high and low $\mathrm{Fe}$ in groundwater ${ }^{(30)}$.

Yet, as anaemia levels remain high in children and women, less than $10 \%$ can be explained by IDA. Other contributors, such as deficiencies in vitamin $B_{6}$, vitamin $B_{12}$, vitamin $A$, vita$\min \mathrm{C}$, folic acid and riboflavin ${ }^{(31)}$, need to be examined, reinforcing the role that other haematopoietic micronutrients play in controlling and preventing $90-95 \%$ of anaemia cases. Reviewing non-nutritional contributors to anaemia (i.e. malaria, worm infestation, chronic infections and genetic disorders (e.g. haemoglobinopathies)) ${ }^{(32-34)}$, it is noted that the risk of malaria is generally low in Bangladesh. While there are no national data on the prevalence of thalassaemia, it is considered a likely contributor, with one study reporting a $28 \%$ prevalence of thalassaemia and an associated risk of anaemia ${ }^{(35)}$.

\section{Zinc deficiency}

The NMS 2011-2012 reports a 44.6 \% prevalence of Zn deficiency among preschool-age children and $57.3 \%$ prevalence in NPNL women, with the highest rate among those living in the slums ${ }^{(11)}$. Zn deficiency was defined by a serum concentration $<9.9 \mathrm{mmol} / \mathrm{l}$ in preschool-age children and a serum concentration $<10.1 \mathrm{mmol} / 1$ in NPNL women according to the International Zinc Consultative Group ${ }^{(36)}$. It is important to note that serum $\mathrm{Zn}$ is homeostatically regulated and unable to detect marginal deficiency; therefore, a high prevalence of low serum $\mathrm{Zn}$ is considered to be a reasonable indicator of a relatively severe deficiency ${ }^{(36)}$. Although there are no national level data on infants and pregnant women, two small-scale studies in rural communities report a $55 \%$ prevalence of $\mathrm{Zn}$ deficiency in pregnant women ${ }^{(37)}$ and a $57 \%$ prevalence of $\mathrm{Zn}$ deficiency in infants ${ }^{(38)}$. Low socio-economic status, household food insecurity, low intake of animal sources of $\mathrm{Zn}$ and high intake of plant-based diet with a very high content of phytate (an inhibitor of $\mathrm{Zn}$ absorption) are the main drivers behind poor $\mathrm{Zn}$ nutrition ${ }^{(15)}$.

\section{Vitamin D deficiency}

Based on a serum vitamin D level $<25 \mathrm{nmol} / 1,7 \cdot 5 \%$ of preschool-age children and $6.5 \%$ of school-age children are deficient in vitamin $\mathrm{D}^{(11)}$. The NMS 2011-2012 indicates that the prevalence of vitamin $\mathrm{D}$ deficiency in preschool-age children is the highest among the poorest and the severely food-insecure 
households; however, for school-age children, the prevalence of vitamin $\mathrm{D}$ deficiency is the highest among the richest and the food-secure households ${ }^{(11)}$. In addition, $32 \cdot 1 \%$ of preschool-age children and $39.0 \%$ of school-age children are living with an insufficient vitamin $\mathrm{D}$ status (i.e. serum vitamin $\mathrm{D}$ $<50.0 \mathrm{nmol} / \mathrm{l}$ ), with the highest prevalence among children living in the slums ${ }^{(11)}$. In 2008, the National Rickets Survey revealed a $1 \%$ prevalence of rickets among children between the ages of 1 and 15 years ${ }^{(39)}$.

The figures for NPNL women suggest that $21 \%$ of these women are deficient based on a serum vitamin $\mathrm{D}$ level $<25.0 \mathrm{nmol} / 1$, and $50 \%$ of them have an insufficient vitamin $\mathrm{D}$ status (serum vitamin D level $<50 \mathrm{nmol} / \mathrm{l})^{(11)}$. No corresponding national-level data are available for pregnant women.

\section{$B$ vitamin deficiencies}

To date, no data are available on the national level estimates of $B$ vitamin deficiencies among infants and children. However, in rural Bangladesh, one earlier small study has shown a 31 $\%$ prevalence of vitamin $\mathrm{B}_{12}$ deficiency in infants ${ }^{(38)}$; whereas another study has shown a $25 \%$ prevalence in folate deficiency, a $7 \%$ prevalence of vitamin $\mathrm{B}_{12}$ deficiency and an $89 \%$ prevalence of vitamin $\mathrm{B}_{2}$ deficiency among adolescent school girls who are anaemic ${ }^{(40)}$.

According to the NMS 2011-2012, $9 \%$ of NPNL women are deficient in folate, and $23 \%$ of these women have some degree of vitamin $\mathrm{B}_{12}$ deficiency ${ }^{(11)}$. One small-scale study has shown a $46 \%$ prevalence of vitamin $B_{12}$ deficiency in women in early pregnancy ${ }^{(37)}$.

\section{Aetiology of micronutrient deficiency}

A large majority of Bangladeshi people follow a diet consisting of predominantly plant-based foods. They have a lack of dietary diversity with a minimum amount of animal food, including eggs, milk and milk products. Thus, a poor-quality diet with poor bioavailability is potentially the major contributor to micronutrient deficiencies in the country ${ }^{(41,42)}$. Although there appears to be an overall increase in the consumption of animal foods in the country $(26.2 \mathrm{~g} /$ capita per $\mathrm{d}$ in $2010^{(43)}$ v. $20 \cdot 8 \mathrm{~g} /$ capita per $\mathrm{d}$ in $\left.2005^{(44)}\right)$, the majority of the population has an inadequate intake for a range of micronutrients. A study on the dietary micronutrient intake among young children and their primary female caregivers in rural Bangladesh indicates a very low overall mean prevalence of adequacy of micronutrient intake, based on estimated average requirements, in children $(43 \%)$ and women $(26 \%)^{(45)}$. For children, the prevalence of adequate intake was $<30 \%$ for $\mathrm{Fe}$, riboflavin and vitamin $\mathrm{B}_{12}$, and it was $<10 \%$ for $\mathrm{Ca}$, folate and vitamin $\mathrm{A}^{(45)}$. A very similar picture was also presented in the NMS 2011-2012 $2^{(11)}$. Furthermore, based on the Household Income and Expenditure Survey 2010, $35 \%$ of the population reportedly has mean dietary diversity scores lower than six out of twelve food groups, and thus, is considered to be at risk of micronutrient deficiency ${ }^{(43)}$.

In addition to a poor-quality diet, the major underlying causes of micronutrient deficiency in the country have been reported to be limited diversity due to low socio-economic status and household food insecurity, low levels of understanding in relation to an optimal diet and hygiene practices, along with infection and infestation ${ }^{(15)}$. It is noteworthy that infectious disease and micronutrient deficiencies exacerbate one another in a vicious cycle. Infections deplete micronutrients, and with limited stores to draw upon, the immune system weakens further and becomes less capable of fighting the infection ${ }^{(46)}$.

\section{Current policies and intervention programmes}

The nature and progress of the current policies and programmes that have been developed to alleviate the problem of micronutrient deficiencies in the country were reviewed to develop the subsequent analysis and discussion related to understanding the persistent deficiencies and moving towards recommendations on new directions. Table 2 shows the current intervention programmes and their coverage rates for prevention and control of micronutrient deficiencies in Bangladesh.

\section{Programmes aimed at improving infant and young child feeding practices}

Through a very wide range of means and methods, the government of Bangladesh, UN agencies, non-governmental organisations and donors have supported interventions aimed at improving infant and young child feeding (IYCF) practices from birth, when exclusive breastfeeding is promoted, through the various stages of the introduction of complementary food from 6 months and beyond. In a number of cases, these interventions are supported together with interventions related to health, food, livelihoods, water, sanitation and hygiene.

\section{Anaemia-control programme for children aged 6-59 months}

The deworming of children aged 24-59 months with albendazole is part of the country's anaemia-control programme for children and is included during the twice-yearly, vitamin A supplementation campaign. In 2011, the national coverage rate for deworming was $77 \%{ }^{(47)}$.

To prevent anaemia and other micronutrient deficiencies, the National Strategy for the Prevention and Control of Anaemia in Bangladesh has recommended using multiple micronutrient powder (MNP) in the diet of children between 6 and 23 months old (and between 24 and 59 months old if resources are available) ${ }^{(48)}$. Currently, there is one large-scale area-based MNP programme in place supported by the Bangladesh Rural Advancement Committee (BRAC)/Global Alliance for Improved Nutrition (GAIN) that uses the government-approved five-component (i.e. Fe, folic acid, $\mathrm{Zn}$ and vitamins A and C) powder. In 2013, the community health workers (Shasthya Shebika) of BRAC distributed 14.5 million sachets of MNP in rural communities through home visits ${ }^{(49)}$.

\section{Vitamin A supplementation}

In settings where VAD is a public health problem, 6-monthly vitamin A supplementation is strongly recommended by the 
Table 2. Current intervention programmes for the prevention and control of various micronutrient deficiencies in Bangladesh

\begin{tabular}{|c|c|c|c|}
\hline Intervention programme & Target group & Current coverage rate & Comments \\
\hline $\begin{array}{l}\text { Vitamin A } \\
\text { supplementation }\end{array}$ & Children under 5 years & $\begin{array}{l}\text { Infants aged } 6-11 \text { months: } \\
85.4 \% \\
\text { Children aged } 12-59 \\
\text { months: } 93.7 \%\end{array}$ & $\begin{array}{l}\text { No remarkable variation in coverage between urban and } \\
\text { rural areas }\end{array}$ \\
\hline $\begin{array}{l}\text { Fortification of edible oil } \\
\text { with vitamin } A\end{array}$ & Whole population & Not available & Recently initiated, no monitoring data available \\
\hline $\begin{array}{l}\text { Fe-folate } \\
\text { supplementation }\end{array}$ & $\begin{array}{l}\text { Pregnant women/ } \\
\text { adolescent girls and non- } \\
\text { pregnant women }\end{array}$ & $\begin{array}{l}\text { Pregnant women: received } \\
\text { IFA through ANC: around } \\
60 \%\end{array}$ & $\begin{array}{l}\text { ANC care is weak - less than } 30 \% \text { of women attend ANC } \\
\text { in the first trimester, and attendance at } 4+\text { visits is around } \\
30 \% \text {. } \\
\text { Inadequate supply at ANC or inadequate knowledge and } \\
\text { practice of the providers - very weak counselling. } \\
\text { Poor monitoring. } \\
\text { No nationwide programme for adolescent girls and non- } \\
\text { pregnant women }\end{array}$ \\
\hline Universal salt iodisation & Whole population & $\begin{array}{l}\text { Adequately iodised salt } \\
\text { (>20 ppm): } \\
\text { Retailer's level: } 66.4 \% \\
\text { Household level: } 57.6 \%\end{array}$ & $\begin{array}{l}\text { Coverage is lowest in rural areas due to the availability of } \\
\text { non-iodised salts which are less costly }\end{array}$ \\
\hline $\begin{array}{l}\text { Home fortification with } \\
\text { MNP }\end{array}$ & Children under 5 years & Adherence to MNP*: $70 \%$ & A small study conducted in an intensive programme area \\
\hline $\begin{array}{l}\text { Promotion of diversified } \\
\text { diet }\end{array}$ & Whole population & N/A & $\begin{array}{l}\text { Policies are in place to improve diversified agriculture, } \\
\text { fisheries and aquaculture and livestock development, and } \\
\text { community-based nutrition programmes, but much } \\
\text { stronger efforts are needed to achieve goal }\end{array}$ \\
\hline
\end{tabular}

IFA, Fe-folic acid; ANC, antenatal care; ppm, parts per million; MNP, multiple micronutrient powder; N/A, not applicable.

${ }^{*}$ Adherence was defined by the consumption of one MNP sachet per $\mathrm{d}$ in the past $60 \mathrm{~d}$.

WHO for infants and children under 5 years of age as a public health intervention to reduce child morbidity and mortality ${ }^{(50)}$. Bangladesh initiated this programme in 1973. Coverage increased from $56 \%$ in $1993^{(51)}$ to $80 \%$ in $1999^{(52)}$ and is reported to be approximately $90 \%$ since $2006^{(53)}$.

While the WHO does not recommend vitamin A supplementation in postpartum women as a public health intervention $^{(54)}$ due to a lack of evidence of its benefits, the Bangladesh government recommends postpartum vitamin $\mathrm{A}$ supplementation. The coverage of postpartum vitamin A supplementation remains low at approximately $36 \%{ }^{(55)}$.

\section{Fortification of edible oil with vitamin A}

Bangladesh initiated the fortification of edible oil with vitamin A in December 2011. Out of twenty-two edible-oil refineries, sixteen produce vitamin A-fortified edible oil. While the government endorsed the 'National Edible Oil Fortification Law 2013', ensuring the $100 \%$ fortification of refined edible oil with vitamin $A$, this programme has not yet reached its targets in relation to coverage ${ }^{(15)}$.

\section{Universal salt iodisation programme}

In Bangladesh, the Universal Salt Iodisation (USI) programme has been in place for more than two decades. By 1999, while nearly $99 \%$ of the edible salts at the factory level were iodised, not all edible salts were adequately iodised according to defined standards ${ }^{(56)}$. The current coverage of adequately iodised salt ( $\geq 20$ parts per million (ppm)) at the retailer level is $66.4 \%$ and at the household level is $57.6 \%{ }^{(11)}$. The coverage is lowest in rural areas. Currently, about a one-quarter of households buy non-iodised bulk salt, and $84 \%$ of those who do not consume iodised salt cite the relatively higher price as the main reason ${ }^{(11)}$.

The USI programme continues to face challenges, including delays in revising the law to include the iodisation of salt destined for the food industry and animals, as per guidance from the $\mathrm{WHO}^{(57)}$. Other significant challenges include insufficient enforcement at the production and retailer level; inadequate information on the actual production of edible and industrial salts; lack of access to packaged, fortified salt in rural and hard-to-reach areas; higher prices of iodised salt; absence of quality control on the importation of edible salt; and overall weak monitoring systems. Despite significant investments in mass communication and targeted information campaigns, consumer and retailer interest remains relatively low ${ }^{(15)}$.

\section{Iron-folic acid supplementation}

While the NMS 2011-2012 indicates overall low Fe deficiency and IDA in children and NPNL women in Bangladesh, an Fe-folic acid (IFA) supplementation programme for pregnant women has been implemented for several decades aimed at preventing and managing Fe deficiency and anaemia. This programme provides IFA supplements $(60 \mathrm{mg}$ Fe and $400 \mu \mathrm{g}$ folic acid daily) to pregnant women from the second trimester until $90 \mathrm{~d}$ after delivery. The IFA supplementation is one of the components of the Health, Population and Nutrition Sector Development Program (HPNSDP). The programme was implemented by the Directorate General of Family Planning (DGFP) and delivered by the Family Welfare Visitors of the DGFP as part of the antenatal care services at the Satellite Clinics and Maternal and Child Welfare Centres. 
However, apart from a number of areas that receive direct support from development partners and non-governmental organisations, coverage remains suboptimal, with a low proportion of women consuming the full recommended dosage. The 2011 report on the State of Food Security and Nutrition in Bangladesh indicated that $37 \%$ of women did not consume any IFA supplements during pregnancy ${ }^{(47)}$, and among those who took the IFA supplements, more than one-half started in the third trimester ${ }^{(47)}$. Coverage rates vary widely by geographical area and by place of residence, with compliance higher among urban-dwelling women. While antenatal care coverage has increased dramatically from $31 \%$ in $1993^{(51)}$ to $79 \%$ in $2014^{(58)}$, only $31 \%$ of pregnant women attended the recommended minimum four visits in 2014. The Bangladesh Health, Population and Nutrition Sector Development Program (HPNSDP) sets a target of $50 \%$ of the pregnant women making at least four visits by $2016^{(59)}$, and clearly Bangladesh is far behind from meeting this target. However, one recent report that conducted an analysis of how well antenatal care clinics are distributing the IFA tablets showed that only $60 \%$ of the pregnant women attended at least one antenatal care, but $26 \%$ of them did not receive any IFA tablets. This could be due to an inadequate supply or inadequate knowledge and practice of the providers ${ }^{(60)}$.

\section{Iron-folic acid supplementation for non-pregnant and non- lactating women and adolescent girls}

In Bangladesh, twice-weekly supplementation with $60 \mathrm{mg} \mathrm{Fe}$ and $400 \mu \mathrm{g}$ folic acid is recommended for the prevention and treatment of IDA in adolescent girls and NPNL women $^{(52)}$. From 2005 to 2010 , a school-based programme in areas outside Dhaka aimed to distribute weekly IFA supplements to adolescent girls. In addition, adolescent girls, newlywed women and postpartum women were provided IFA supplements and anthelmintic treatment in areas covered by the National Nutrition Programme. In 2011, the National Nutrition Programme was replaced by the National Nutrition Services as one of the operational plans under the Health, Population and Nutrition Sector Development Program (HPNSDP) and implemented by the Institute of Public Health Nutrition to ensure the mainstreaming of nutrition. While the National Nutrition Services has established a guideline for IFA supplementation for NPNL women and adolescent girls, currently there is no nationwide IFA supplementation programme or platform to reach this target group.

The current IFA supplementation programme has several strengths that include a government-approved national anaemia strategy, IFA distribution guidelines and an integrated health and family planning wing under the National Nutrition Services operational plan. However, one major weakness is the lack of a detailed plan of action (roadmap), along with inadequate coordination and monitoring between the Ministry of Health and Family Welfare and other non-governmental organisations that are also delivering IFA tablets through their programmes ${ }^{(60)}$. An analysis of the bottlenecks leading to the inadequacy of effective coverage in relation to IFA supplementation undertaken by UNICEF in 2012-2013 showed that very specific issues needed to be addressed relating to supply, uptake of services and compliance with treatment at the household level. Specific targets for IFA supplementation are often absent and not adequately covered by certain indicators, such as attendance for antenatal care (UNICEF, Dhaka: unpublished results). Counselling and follow-up related to compliance with supplementation regimens are not routinely included in care protocols.

The level of education of the beneficiaries and lack of awareness about the benefit of IFA supplements are some of the key contributing factors to the low coverage rate ${ }^{(15)}$. Ensuring a consistent and adequate supply of IFA tablets and introduction of indicators and targets related to actual bottlenecks will be required to address the current inadequate coverage.

\section{Promotion of diversified diet}

Throughout Bangladesh, many government and non-government entities have supported a wide variety of approaches and programmes aimed at increasing the diversity and quality of the diet. The Bangladesh Country Investment Plan is described as a 'roadmap towards investment in agriculture, food security and nutrition', and identified twelve programmes as part of its Plan of Action 2008-2015 $5^{(61)}$. The Bangladesh Country Investment Plan supports community-based homestead gardening, rearing small livestock, aquaculture and awareness building, aiming to increase household availability and access to food in general, but especially micronutrientrich foods. The 2011-2012 food security nutritional surveillance programme report estimated that $42 \%$ of households had both homestead gardens and poultry, $14 \%$ had homestead gardens alone, $20 \%$ had poultry and $24 \%$ had neither ${ }^{(47)}$.

To optimise the potential of these programmes to address micronutrient deficiencies, targeting, outcome monitoring and stronger communication interventions will be required. Although the National Nutrition Services supports mass media campaigns, social mobilisation and behaviour change communication activities at the health facility and community clinic levels, these specific nutrition-related messages also need to be reinforced through the Ministry of Agriculture and other food-related ministries. Opportunities exist to create and strengthen markets for nutritious foods, as well as to invest in value chain and postharvest technologies that can help retain and enhance the nutritional content of foods.

\section{Programme implications}

Using the findings of the NMS 2011-2012 and complementary data, the government and partners developed the 'National Strategy on Prevention and Control of Micronutrient Deficiencies, Bangladesh (2015-2024)', which was approved in May $2015^{(15)}$. This strategy has laid out a very comprehensive action plan divided into six strategic areas: (i) policy, guidelines and legislation; (ii) intervention programmes; (iii) partnerships and coordination; (iv) capacity building; (v) advocacy and communication; and (vi) monitoring, evaluation and research ${ }^{(15)}$. 
In this strategy document, a number of key interventions are recommended for controlling micronutrient deficiencies. Some of these interventions have been implemented for many years and mainly require strengthening, while other interventions are new in the country and require adaptation to country context and system development. These interventions are divided into three broad categories. The first is ensuring that all individuals, throughout their life-cycle, have a fully adequate nutrient intake, and maintain optimal health, through optimal infant and young child feeding (IYCF) and improved dietary diversification. Second, to complement diet- and carebased approaches, evidence-based and cost-effective food fortification is recommended, with the provision that the fortified foods must reach those who most need them. Finally, to address the immediate requirements of individuals and populations most at risk or already experiencing the debilitating and life-threatening effects of micronutrient deficiencies, targeted supplementation is recommended.

\section{Discussion}

The most recent national survey, NMS 2011-2012, has provided critical information to inform decision making and programming related to public health and nutrition in Bangladesh. It has also presented a number of challenges at both the technical and operational levels. While the country moved ahead to develop a new strategy, a number of issues are worthy of further discussion, analysis and research.

First, it is noteworthy that despite very high coverage of a 6-monthly vitamin A capsule supplementation programme for children aged 6-59 months in the country, currently one in five children are living with VAD, with the highest prevalence among children living in the slums. Several studies have demonstrated that a 6-monthly high-dose vitamin A capsule can only achieve a transient and small increase in serum retinol that could sustain the level for about 2 months. Therefore, this programme is unable to protect against mild-moderate VAD (serum retinol $<0.70 \mu \mathrm{mol} / \mathrm{l}$ ) up to 6 months, although it can protect the eyes from clinical $\mathrm{VAD}^{(62,63)}$. A recent article by Mason et al. ${ }^{(64)}$ indicated that frequent intakes of vitamin $\mathrm{A}$ in physiological doses either through regular low-dose supplementation or through foodbased approaches, including fortification, can be highly effective in increasing serum retinol, thus reducing VAD. The authors emphasised the need of a policy shift from periodic vitamin A capsules to increasing frequent regular intakes of vitamin $\mathrm{A}$ at physiological levels ${ }^{(64)}$. However, the replacement of vitamin A capsules with a food-based strategy is not an immediately feasible option in the absence of access to adequate vitamin A-rich food, including fortified food for those who are vulnerable.

Second, recent findings on the association between the natural Fe content of groundwater and Fe status have introduced new challenges for public health programming. It is evident that there is variability in the prevalence of Fe deficiency across the country due to varied Fe levels in groundwater ${ }^{(30,65)}$. This may have serious implications for a future IFA supplementation programme for controlling anaemia and Fe deficiency in pregnant women in the country and thus there is an urgent need for reviewing the current IFA supplementation guidelines. However, it is important to recognise the fact that the need for Fe increases significantly during pregnancy to meet the demand of physiological changes and fetal development ${ }^{(66)}$. Based on the data in NPNL women, it is difficult to predict whether or not Bangladeshi pregnant women living in areas of either high or low $\mathrm{Fe}$ in groundwater can maintain a satisfactory Fe status, which is absolutely critical for fetal development, throughout pregnancy without IFA supplementation. On the other hand, it is also equally important to know whether or not IFA supplementation in this population, especially those living in areas of high $\mathrm{Fe}$ in groundwater, can cause Fe overload. Fe is a strong pro-oxidant and high body Fe levels have been linked to oxidative stress that in turn has been implicated in atherosclerosis, CVD, diabetes and the metabolic syndrome ${ }^{(67,68)}$. Elevated Fe stores are also associated with the exaggeration of systemic infections ${ }^{(69)}$. Further, excess dietary intake of $\mathrm{Fe}$ has been found to be associated with increased growth and virulence of enteric pathogens which in turn increase gut inflammation ${ }^{(70,71)}$. IFA supplementation during pregnancy is a key direct nutrition intervention that UNICEF is supporting the government to scale-up to effective coverage; however, given the recent findings of the NMS 2011-2012, the relevancy of this intervention needs further investigation. The persistence of non-Fe-related anaemia also requires further understanding.

Third, given the variability in the prevalence of Fe deficiency and IDA in the country based on the most recent micronutrient survey, MNP programmes for children aged 6-59 months should be carefully evaluated to see if there is a need for change in the composition of MNP.

Fourth, the USI programme in Bangladesh provides a good example of an area where we know exactly what needs to be done; however, despite the investment of significant resources and an apparent good commitment for implementation, achievement has been lower than expected. This might also be a moment to comment on the fact that while many programmes are consistently increasing their coverage (in numbers), it is not adequately intensive to keep pace with the rapid population growth; therefore, percentages remain stagnant.

Fifth, it is important to note that the current prevalence of $\mathrm{Zn}$ deficiency is much higher than VAD in children and NPNL women in the country. Currently, the Bangladesh government has implemented an adjunctive treatment of $\mathrm{Zn}$ with oral rehydration in diarrhoea for young children, which is independent of the underlying $\mathrm{Zn}$ status of the population. Thus, there is a need to consider the appropriate intervention for the prevention of $\mathrm{Zn}$ deficiency in the country.

Finally, in Bangladesh and globally, we have a debate related to short/medium-term approaches $v$. long-term approaches to address micronutrient deficiencies. It will be important in the months and years ahead to monitor the situation closely, ensuring that the appropriate mix of interventions is supported using evidence-based approaches. Approaches that address immediate threats to wellbeing should not be seen to compete with meaningful interventions to ensure the long-term dietary 
adequacy of the population. It will be increasingly important to identify specific nutrition-related indicators in food-related programmes, ensuring that interventions first understand and subsequently address specific dietary deficits, be they seasonal or for specific groups of people (e.g. age or wealth group). In the short term and to meet the specific needs of vulnerable groups, agriculture and horticulture programmes that enhance diet quality and diversity must be combined with supplementation programmes specifically designed to address dietary deficits.

\section{Conclusion}

This review illustrates that while the severity of many micronutrient deficiencies in Bangladesh has decreased remarkably over the past few decades, a significant proportion of the population, especially children and women, is still deficient in critical micronutrients. Several intervention programmes are in place to alleviate the problem of micronutrient deficiencies, especially for vitamin A, Fe and I deficiency. However, the success of these interventions is far from satisfactory. Given the complex nature of factors contributing to micronutrient deficiencies in Bangladesh, like other low-income countries, the prevention and control of micronutrient deficiency in the country are unlikely to be achieved through nutrient-specific nutrition policies and programmes. More holistic approaches that can integrate into short- to long-term strategies are necessary to achieve and maintain sustainable improvement of the present situation. It is also essential that there are sufficient resources and collaboration between health and food sector workers, which is vital for ensuring quality service delivery and accurate monitoring and reporting for improved outcomes in relation to the micronutrient status of targeted groups. Finally, advocacy is needed to influence government policy across core sectors in combating micronutrient malnutrition through support from the highest level of leadership.

\section{Acknowledgements}

The authors gratefully acknowledge Ireen Chowdhury (UNICEF, Dhaka); Institute of Public Health Nutrition, Ministry of Health and Family Welfare, Dhaka, Bangladesh; International Centre for Diarrhoeal Disease Research, Bangladesh; Micronutrient Initiative, Dhaka; Food and Agricultural Organization, Dhaka; Global Alliance for Improved Nutrition, Dhaka; and Institute of Nutrition and Food Science, University of Dhaka, Dhaka, Bangladesh for providing us the valuable information.

This research received no specific grant from any funding agency, commercial or not-for-profit sectors.

F. A. designed the concept of the study, conducted the literature review and analysis, and prepared the manuscript draft. N. P. and A. N. critically reviewed the manuscript and contributed in writing. All authors read and approved the final manuscript.

There were no competing interests.

\section{References}

1. United Nations Children's Fund, Micronutrient Initiative (2004) Vitamin and Mineral Deficiency: A Global Progress Report. Ottawa, Canada: Micronutrient Initiative.

2. Huffman SL, Baker J, Shumann J, et al. (1999) The Case for Promoting Multiple Vitamin/Mineral Supplements for Women of Reproductive Age in Developing Countries. Washington, DC: Linkages Project, Academy for Educational Development.

3. Black RE, Allen L, Bhutta AZ, et al. (2008) Maternal and child undernutrition: global and regional exposures and health consequences. Lancet 371, 243-260.

4. Hetzel BS (1983) Iodine deficiency disorders and their eradication. Lancet ii, 1126-1129.

5. Haas JD \& Brownlie T (2001) Iron deficiency and reduced work capacity: a critical review of the research to determine a causal relationship. J Nutr 131, Suppl., 676-678.

6. Horton S \& Ross J (2003) The economics of iron deficiency. Food Policy 28, 51-75.

7. Imdad A, Herzer K, Mayo-Wilson E, et al. (2010) Vitamin A supplementation for preventing morbidity and mortality in children from 6 months to 5 years of age. Cocbrane Database Syst Rev 12, CD008524.

8. Yakoob MY, Theodoratou E, Jabeen A, et al. (2011) Preventive zinc supplementation in developing countries: impact on mortality and morbidity due to diarrhea, pneumonia and malaria. BMC Public Health 11, Suppl. 3, S3-S23.

9. Bhutta ZA, Das JK, Rizvi A, et al. (2013) Evidence-based interventions for improvement of maternal and child nutrition: what can be done and at what cost? Lancet 382, 452-477.

10. Government of the People's Republic of Bangladesh (2014) Millennium Development Goals: Bangladesh Country Report 2013. Dhaka, Bangladesh: Planning Commission, Government of the People's Republic of Bangladesh.

11. International Centre for Diarrhoeal Diseases Research, Bangladesh (ICDDR,B), Global Alliance for Improved Nutrition (GAIN), The United Nations Children's Fund (UNICEF) (2013) The National Micronutrients Status Survey 2011-12. Dhaka, Bangladesh: International Centre for Diarrhoeal Diseases Research, Bangladesh.

12. Institute of Public Health Nutrition (IPHN), Helen Keller International (HKI) (1985) Bangladesh Nutritional Blindness Study 1982-83. Dhaka, Bangladesh: IPHN and HKI.

13. Helen Keller International (HKI), Institute of Public Health Nutrition (IPHN) (2005) Bangladesh in Facts and Figures 2004: Annual Report of the Nutritional Surveillance Project. Dhaka, Bangladesh: HKI and IPHN.

14. Helen Keller International (HKI), Institute of Public Health Nutrition (IPHN) (1999) Vitamin A Status Throughout the Life Cycle in Rural Bangladesh, Bangladesh National Vitamin A Survey, 1997-98. Dhaka, Bangladesh: HKI and IPHN.

15. Institute of Public Health Nutrition (2015) National Strategy on Prevention and Control of Micronutrient Deficiencies, Bangladesh (20152024). Dhaka, Bangladesh: Institute of Public Health Nutrition, Ministry of Health and Family Welfare, Government of the People's Republic of Bangladesh.

16. Dhaka University, Institute of Public Health Nutrition (IPHN), Bangladesh Small and Cottage Industries Corporation (BSCIC), The United Nations Children's Fund (UNICEF), International Council for the Control of Iodine Deficiency Disorders (ICCIDD) (1999) Report of the National Iodine Deficiency Survey of Bangladesh, 1999. Dhaka, Bangladesh: Dhaka University/IPHN/ BSCIC/UNICEF/ICCIDD.

17. Dhaka University, Institute of Public Health Nutrition (IPHN), Bangladesh Small and Cottage Industries Corporation (BSCIC), The United Nations Children's Fund (UNICEF), International Council for the Control of Iodine Deficiency Disorders (ICCIDD) (2007) National Survey on Iodine Deficiency Disorders and Universal Salt Iodization in Bangladesh 2004-5. Dhaka, Bangladesh: Dhaka University/IPHN/BSCIC/UNICEF/ICCIDD. 
18. Dhaka University, International Council for the Control of Iodine Deficiency Disorders (ICCIDD), The United Nations Children's Fund (UNICEF) (1993) Report of the National Iodine Deficiency Survey of Bangladesh, 1993. Dhaka, Bangladesh: Dhaka University/ IPHN/ICCIDD/UNICEF.

19. Helen Keller International (HKI), Institute of Public Health Nutrition (IPHN) (1999) Iron deficiency Anaemia Throughout the Life Cycle in Rural Bangladesh. Dhaka, Bangladesh: HKI and IPHN.

20. Bangladesh Bureau of Statistics (2004) Anaemia Prevalence Survey of Urban Bangladesh and Rural Chittagong Hill Tracts 2003. Dhaka, Bangladesh: Ministry of Planning, Government of the People's Republic of Bangladesh.

21. National Institute of Population Research and Training (NIPORT), Mitra and Associates, ICF International (2013) Bangladesh Demographic and Health Survey Report 2011. Dhaka, Bangladesh and Rockville, MD: NIPORT, Mitra and Associates, and ICF International.

22. Ahmed F, Hasan N \& Kabir Y (1997) Vitamin A deficiency among adolescent garment factory workers in Bangladesh. Eur J Clin Nutr 51, 698-702.

23. Helen Keller International (HKI), Institute of Public Health Nutrition (IPHN) (2002) Anaemia is a Serious Public Health Problem in Pre-school Children and Pregnant Women in Rural Bangladesh. Nutrition Surveillance Project Bulletin No. 10. Dhaka, Bangladesh: HKI and IPHN.

24. Helen Keller International (HKI), Institute of Public Health Nutrition (IPHN) (2006) The Burden of Anaemia in Rural Bangladesh: An Urgent Need for Action. Nutrition Surveillance Project Bulletin No. 16. Dhaka, Bangladesh: HKI and IPHN.

25. Ahmed F, Khan MR, Islam M, et al. (2000) Anaemia and iron deficiency among adolescent schoolgirls in peri-urban Bangladesh. Eur J Clin Nutr 54, 678-683.

26. Ahmed F, Khan MR \& Jackson AA (2001) Concomitant supplementation with vitamin A enhances the response to weekly supplemental iron and folic acid in aaemic teenage women in urban Bangladesh. Am J Clin Nutr 74, 108-115.

27. Merrill RD, Labrique AB, Shamim AA, et al. (2010) Elevated and variable groundwater iron in rural north western Bangladesh. $J$ Water Health 8, 818-825.

28. Hallberg L (1991) Bioavailability of dietary iron in man. Ann Rev Nutr 1, 123-147.

29. Merrill RD, Shamim AA, Ali H, et al. (2011) Iron status of women is associated with the iron concentration of potable groundwater in rural Bangladesh. J Nutr 141, 944-949.

30. Rahman S, Ahmed T, Rahman AS, et al. (2016) Determinants of iron status and $\mathrm{Hb}$ in the Bangladesh population: the role of ground water iron. Public Health Nutr 19, 1862-1874.

31. Fishman SM, Christian P, West KP Jr (2000) The role of vitamins in the prevention and control of anaemia. Public Health Nutr 3, 125-150.

32. Dreyfuss ML, Stoltzfus RJ, Shrestha JB, et al. (2000) Hookworm, malaria and vitamin A deficiency contribute to anemia and iron deficiency among pregnant women in the plains of Nepal. J Nutr 130, 2527-2536.

33. Jansson LT, Kling S \& Dallman PH (1986) Anemia in children with acute infection seen in a primary care pediatric outpatient clinic. Paediatr Infect Dis 5, 424-427.

34. Linpisarn S, Tienboon P, Promtet N, et al. (1996) Iron deficiency and anemia in children with a high prevalence of haemoglobinopathies: implications for screening. Int J Epidemiol 25, 1262-1266.

35. Merrill RD, Shamim AA, Ali H, et al. (2012) High prevalence of anemia with lack of iron deficiency among women in rural Bangladesh: a role for thalassemia and iron in groundwater. Asia Pac J Clin Nutr 21, 416-424.

36. International Zinc Nutrition Consultative Group (IZiNCG), Brown $\mathrm{KH}$, Rivera JA, et al. (2004) International Zinc Consultative Group (IZiNCG) technical document \# 1. Assessment of the risk of zinc deficiency in populations and options for its control. Food Nutr Bull 25, Suppl. 2, S99-S203.
37. Lindström E, Hossain MB, Lönnerdal BO, et al. (2011) Prevalence of anemia and micronutrient deficiencies in early pregnancy in rural Bangladesh, the MINIMat trial. Acta Obstet Gynecol Scand 90, 47-56.

38. Eneroth H, Arifeen SL, Persson L-A, et al. (2010) Maternal multiple micronutrient supplementation has limited impact on micronutrient status of Bangladeshi infants compared with standard iron folic acid supplementation. J Nutr 140, 618-624.

39. Kovacs CS (2008) Vitamin D in pregnancy and lactation: mineral, fetal, and neonatal outcomes from human and animal studies. Am J Clin Nutr 88, Suppl., S520-S528.

40. Ahmed F, Khan MR, Banu CP, et al. (2008) The coexistence of other multiple micronutrient deficiencies in anaemic adolescent schoolgirls in rural Bangladesh. Eur J Clin Nutr 62, 365-372.

41. Jahan K \& Hossein M (1998) Bangladesh National Nutrition Survey, 1995-96. Dhaka, Bangladesh: Institute of Nutrition and Food Science, University of Dhaka.

42. Ahmed F, Zareen M, Khan MR, et al. (1998) Dietary pattern, nutrient intake and growth of adolescent school girls in urban Bangladesh. Public Health Nutr 1, 83-92.

43. Bangladesh Bureau of Statistics (2010) Report of the Household Income and Expenditure Survey: 2010. Dhaka, Bangladesh: Planning Division, Ministry of Planning, Government of the People's Republic of Bangladesh.

44. Bangladesh Bureau of Statistics (2005) Report of the Household Income and Expenditure Survey: 2005. Dhaka, Bangladesh: Planning Division, Ministry of Planning, Government of the People's Republic of Bangladesh.

45. Arsenault JE, Yakes EA, Islam MM, et al. (2013) Very low adequacy of micronutrient intakes by young children and women in rural Bangladesh is primarily explained by low food intake and limited diversity. J Nutr 143, 197-203.

46. Darnton-Hill I, Ahmed F \& Samon S (2015) The impact of micronutrients on inflammation and health in low- and middle-income countries. In Preventive Nutrition: The Comprehensive Guide for Health Professionals, 5th ed., chapter 30, pp. 597-644 [A Bendich and RJ Deckelbaum, editors]. New York: Springer.

47. James P, Grant School of Public Health (JPGSPH) and Helen Keller International (HKI) (2012) State of Food Security and Nutrition in Bangladesh, 2011. Dhaka, Bangladesh: JPGSPH and HKI.

48. Institute of Public Health Nutrition (IPHN) (2007) National Strategy for Anaemia Prevention and Control. Dhaka, Bangladesh: IPHN.

49. Afsana K, Haque MR, Sobhan S, et al. (2014) BRAC's experience in scaling-up MNP in Bangladesh. Asia Pac J Clin Nutr 23, 377-384.

50. World Health Organization (2003) Guideline: Vitamin A Supplementation in Infants and Children 6-59 Months of Age. Geneva: WHO.

51. National Institute of Population Research and Training (NIPORT), Mitra Associates, Macro International (1993) Bangladesh Demographic and Health Survey Report, 1993. Dhaka, Bangladesh and Calverton, MD: NIPORT, Mitra Associates, and Macro International.

52. National Institute of Population Research and Training (NIPORT), Mitra Associates, Macro International (1999) Bangladesh Demographic and Health Survey Report, 1999. Dhaka, Bangladesh and Calverton, MD: NIPORT, Mitra Associates, and Macro International.

53. Directorate General of Health Services (2014) EPI Coverage Evaluation Survey, 2014. Dhaka, Bangladesh: Ministry of Health and Family Welfare, Government of the People's Republic of Bangladesh.

54. World Health Organization (2011) Guidelines: Vitamin A Supplementation in Postpartum Women. Geneva: WHO.

55. Directorate General of Health Services (2012) EPI Coverage Evaluation Survey, 2012. Dhaka, Bangladesh: Ministry of Health and Family Welfare, Government of the People's Republic of Bangladesh.

56. Institute of Public Health Nutrition (IPHN), Bangladesh Small and Cottage Industries Corporation (BSCIC), The United Nations Children's Fund (UNICEF), International Council for the Control of Iodine Deficiency Disorders (ICCIDD) (1999) 
Evaluation of Universal Salt Iodization (USI) in Bangladesh, 1999. Dhaka, Bangladesh: Dhaka University/IPHN/ICCIDD/UNICEF.

57. World Health Organization, The United Nations Children's Fund (UNICEF), International Council for the Control of Iodine Deficiency Disorders (ICCIDD) (2007) Assessment of Iodine Deficiency Disorders and Monitoring their Elimination: A Guide for Programme Managers. Geneva: WHO.

58. National Institute of Population Research and Training (NIPORT), Mitra and Associates, ICF International (2015) Bangladesh Demographic and Health Survey Report 2014: Key Indicators. Dhaka, Bangladesh and Rockville, MD: NIPORT, Mitra and Associates, and ICF International.

59. Ministry of Health and Family Welfare (2011) Health, Population and Nutrition Sector Development Program (HPNSDP): July 2011-June 2016. Programme Implementation Plan (PIP), vol. 1. Dhaka, Bangladesh: Ministry of Health and Family Welfare, Government of the People's Republic of Bangladesh.

60. Fiedler J, D'Agostino A \& Sununtnasuk C (2014) Nutrition Technical Brief: A Rapid Initial Assessment of the Distribution and Consumption of Iron-Folic Acid Tablets Through Antenatal Care in Bangladesh. Arlington, VA: USAID/Strengthening Partnerships, Results and Innovations in Nutrition Globally (SPRING) Project.

61. Food Planning and Monitoring Unit (2011) Bangladesh Country Investment Plan: A Road Map Towards Investment in Agriculture, Food Security and Nutrition. Dhaka, Bangladesh: Ministry of Food and Disaster Management, Government of the People's Republic of Bangladesh.
62. Solon F, Fernandez TL, Latham MC, et al. (1979) An evaluation of strategies to control vitamin A deficiency in the Philippines. Am J Clin Nutr 32, 1445-1453.

63. Pereira SM \& Begum A (1971) Failure of a massive single oral dose of vitamin A to prevent deficiency. Arch Dis Child 46, 525-527.

64. Mason J, Greiner T, Shrimpton R, et al. (2015) Vitamin A policies need rethinking. Int J Epidemiol 44, 283-292.

65. British Geological Survey \& Department of Public Health Engineering, Government of the People's Republic of Bangladesh (2001) Arsenic Contamination of Groundwater in Bangladesh. BGS Technical Report WC/00/19 [DG Kinniburgh and PL Smedley, editors]. Keyworth: BGS.

66. World Health Organization (2012) Guideline: Daily Iron and Folic Acid Supplementation in Pregnant Women. Geneva: WHO.

67. Jomova $\mathrm{K} \&$ Valko M (2011) Advances in metal-induced oxidative stress and human diseases. Toxicology 283, 65-87.

68. Jehn M, Clark JM \& Guallar E (2004) Serum ferritin and risk of metabolic syndrome in U.S. adults. Diabetes Care 27, 2422-2428.

69. Cassat JE \& Skaar EP (2013) Iron in infection and immunity. Cell Host Microbe 13, 509-519.

70. Zimmermann MB, Chassard C, Rohner F, et al. (2010) The effect of iron fortification on the gut microbiota in African children: a randomized control trial in Cote d'Ivoire. Am J Clin Nutr 92, 14061415.

71. Kortman GAM, Raffatellu M, Swinkels DW, et al. (2014) Nutritional iron turned inside out: intestinal stress from a gut microbial perspective. FEMS Microbiol Rev 38, 1202-1234. 